\title{
ESTIMATION OF THE INTENSIVELY BLASTED ELECTRIC ARC MODEL SENSITIVITY TO SELECTED VARIABLES
}

\author{
J. Senk*, I. Laznickova, I. Jakubova, O. Coufal \\ Faculty of Electrical Engineering and Communication, BUT, Technicka 12, 61600 Brno, Czech Republic \\ * senk@feec.vutbr.cz
}

\begin{abstract}
Results of measurements carried out on the fabricated experimental modular-type arc heater serve as input data for the designed simplified model of the intensively blasted electric arc burning in argon inside the cylindrical arc heater's anode channel. The axial dependence of the arc temperature and radius is expressed using the exponent, the current density on the cathode tip and the arc temperature at the end of the near-cathode boundary layer. These quantities form the vector of state variables that is sought to minimize the value of the objective function expressing the deviations between measured and computed values. On a typical example, the paper demonstrates the sensitivity of the modelling to individual state variables.
\end{abstract}

Keywords: electric arc, arc heater, argon, modeling.

\section{Introduction}

In the experimental modular-type arc heater, which has been designed and fabricated at the Department of Electrical Power Engineering of the Faculty of Electrical Engineering and Communication, Brno University of Technology for both educational and research purposes, the electric arc is burning in the cylindrical anode channel with water-cooled walls and is stabilized by intensive flow of argon (see Fig. 1 of [1]). For each experiment, a set of measured values is recorded, including the arc current, the total voltage, the gas flow rate, and power loss in individual mutually electrically and thermally isolated segments of the arc heater (cathode - subscript cat, input part of the anode channel - subscript in, the anode channel itself subscript ch, the grounded anode - subscript an).

A simple model of the intensively blasted electric arc inside the anode channel has been designed to describe the behavior of the arc and to reveal mutual relationships between main arc heater's operational quantities and arc's properties in a simple and clear form suitable for educational purposes. The model is based on the mass and energy conservation laws and Ohm's law and includes several simplifying presumptions (e.g. LTE, cylindrical symmetry, rectangular arc temperature profile, etc.) $[2,3]$. Measured values together with transport and thermodynamic properties of argon [4-6] serve as input data of the mathematical model.

\section{The Vector of State Variables and the Objective Function}

The behavior of the arc in the anode channel is characterized by the axial development of the arc temperature $T_{\mathrm{A}}(z)$ and arc radius $r_{\mathrm{A}}(z)$. A rectangular temperature profile across the anode channel crosssection is assumed, with the arc zone $T_{\mathrm{A}}(r, z)=T_{\mathrm{A}}(z)$ surrounded by cold argon. The dependence of the arc radius $r_{\mathrm{A}}(z)$ on the axial coordinate $z$ is defined by the exponent $n_{\mathrm{r}}$ and by the cathode spot radius $r_{0}$ corresponding to the current density on the cathode tip $j_{0}$ as follows [7]

$$
r_{\mathrm{A}}(z)=r_{0}\left[1+\left(\frac{z}{r_{0}}\right)^{\left(1 / n_{\mathrm{r}}\right)}\right] .
$$

The behavior of the arc in the close vicinity of the cathode is characterized by the width of the nearcathode boundary layer $s$ and by the temperature $T_{\mathrm{A}}(s)$ there [2].

These three quantities $\left[n_{\mathrm{r}} ; j_{0} ; T_{\mathrm{A}}(s)\right]$ form the vector of state variables that is sought for to minimize the value of the objective function $\Delta_{\text {tot }}$. The objective function $\Delta_{\text {tot }}$ is given by the deviations between the measured and computed values of the arc power loss in individual segments of the device and the voltage drop along the arc

$$
\Delta_{\mathrm{tot}}=\sqrt{\Delta_{\mathrm{in}}^{2}+\Delta_{\mathrm{ch}}^{2}+\Delta_{\mathrm{U}}^{2}} .
$$

The first term $\Delta_{\text {in }}$ describes the relative deviation between the measured (subscript $\mathrm{m}$ ) and calculated (subscript c) values of the arc power loss in the input part of the arc heater, and similarly, the second term $\Delta_{\text {ch }}$ does for the main part of the anode channel

$$
\Delta_{\text {in }}=\frac{P_{\text {loss_in_c }}}{P_{\text {loss_in_m }}}-1, \Delta_{\text {ch }}=\frac{P_{\text {loss_ch_c }}}{P_{\text {loss_ch_m }}}-1 .
$$

The measured power loss is determined from the cooling water flow rate in the corresponding segment and the increase of its temperature. The computed arc power loss is calculated using the theoretically determined values of the net emission coefficient of argon which is used as the working gas in the discussed experiments $[1,6,8]$. 
The third term $\Delta_{\mathrm{U}}$ expresses the relative deviation between the measured and calculated values of the arc voltage drop. As neither the arc voltage drop over the whole $\operatorname{arc} U_{\mathrm{A}}\left(z_{\mathrm{L}}\right)$ nor the power consumed for an arc-anode attachment can be measured separately, this last term compares the arc power $U_{\mathrm{A}}\left(z_{\mathrm{L}}\right) I_{\mathrm{m}}$ decreased by the measured power loss of the cathode $P_{\text {loss_cat_m }}$ and the computed power loss of the arcanode attachment $P_{\text {loss_as_c }}$ versus the total input power of the device $U_{\mathrm{m}} \bar{I}_{\mathrm{m}}$ as follows

$$
\Delta_{\mathrm{U}}=\frac{U_{\mathrm{A} \_\mathrm{c}}\left(z_{\mathrm{L}}\right) I_{\mathrm{m}}+P_{\text {loss_cat_m }}+P_{\text {loss_as_c }}}{U_{\mathrm{m}} I_{\mathrm{m}}}-1 .
$$

More details on the model and its gradual refinement can be found in [1-3]. Here, only the selected formulas and their short explanation is repeated to make the following discussion easy to understand.

\section{Results and Discussion}

The paper focuses on the sensitivity of the objective function to the state variables. The investigation aims at making the search of the optimum state variables' vector as effective as possible. Although some preliminary considerations on sensitivity of the model to individual state variables can be derived, qualitative knowledge about how significantly the sensitivity is changing in what a range must be obtained using concrete experimental data.

In the following, the dependence of the objective function as a whole and of its components on the state variables' vector $\left[n_{\mathrm{r}} ; j_{0} ; T_{\mathrm{A}}(s)\right]$ is shown for a chosen experiment. The experimental arrangement of the arc heater is the following: the anode channel radius $r_{\mathrm{c}}=8 \mathrm{~mm}$, the anode channel length $z_{\mathrm{L}}=109$ $\mathrm{mm}$ (the input part $22 \mathrm{~mm}$, the main anode channel $60 \mathrm{~mm}$, the grounded anode $27 \mathrm{~mm}$ ). The arc was burning in argon with flow rate of $22.5 \mathrm{~g} / \mathrm{s}$. The arc current was set to $122.0 \mathrm{~A}$ and the total voltage drop over the arc heater was $111.8 \mathrm{~V}$. The total input power was $13.64 \mathrm{~kW}$ and the total measured power loss was $3.23 \mathrm{~kW}$. The chosen experiment corresponds to the middle slightly increasing region of the arc-voltage characteristic of the device where the arc exhibits a good stability.

The computation was made for $n_{\mathrm{r}}$ around the value of 3 given by other authors [7], with a step of 0.1 which can be decreased, especially near the minimum of the objective function. For each $n_{\mathrm{r}}$, the calculation was made in a mesh of $j_{0}$ (up to $4 \times 10^{8} \mathrm{Am}^{-2}$, step $0.01 \times 10^{8} \mathrm{Am}^{-2}$ ) and $T_{\mathrm{A}}(s)$ (between $6000 \mathrm{~K}$ up to approx. $18000 \mathrm{~K}$, step $10 \mathrm{~K}$ ) and the minimum was found for this $n_{\mathrm{r}}$. The absolute minimum value of the objective function $\Delta_{\text {tot_min }}=0.03705$ has been reached for $n_{\mathrm{r} \_ \text {min }}=2.45, j_{0 \_ \text {min }}=2.93 \times 10^{8} \mathrm{Am}^{-2}$ and $T_{\mathrm{A}}(s)_{\min }=13400 \mathrm{~K}$.

Figure 1 shows the dependence of the computed minimum values of the objective function $\Delta_{\text {tot }}$ and the corresponding values of $j_{0}$, the cathode boundary layer width $s$, the arc temperature $T_{\mathrm{A}}(s)$ and the electric field intensity $E(s)$, both at the end of the boundary layer, and the arc temperature $T_{\mathrm{A}}\left(z_{1}\right)$ at the first computational step $z_{1}=1 \mathrm{~mm}$ from the cathode tip, on the exponent $n_{\mathrm{r}}$. Mutual correspondence of temperatures, near-cathode boundary layer width $s$ and $E(s)$ was discussed in previous works [3] and will be mentioned later. Obviously, in the investigated case, the found minimum values of $\Delta_{\text {tot }}$ first quickly decrease with increasing $n_{\mathrm{r}}$ and after reaching the global minimum value $\Delta_{\text {tot_min }}=3.705 \%$ for $n_{\mathrm{r} \_ \text {min }}=2.45$ they start to increase slowly.

Figure 2 shows again the computed minimum values of the objective function $\Delta_{\text {tot }}$ but in dependence on other state variables $\left(j_{0}, T_{\mathrm{A}}(s)\right)$ and altogether with the other two state variables. While a pronounced global minimum for exponent $n_{\mathrm{r} \_ \text {min }}=2.45$ can be seen in Fig. 1, in Fig. 2 the computed minimums of the objective function exhibit significant changes for low and high values of both $j_{0}$ and $T_{\mathrm{A}}(s)$, but preserve only slightly changing values in a rather wide middle region. This observation confirms the leading role of the exponent $n_{\mathrm{r}}$.

As explained in our previous work [3] also some other points of view should be taken into account to obtain physically acceptable results. More detailed analysis can show that for some obtained minimums of the objective function, the computed arc temperature $T_{\mathrm{A}}(z)$ tends to oscillate, especially near the beginning. Another problem to be avoided is an extremely high electric field intensity at the end of the cathode boundary layer and consequently an extremely thin boundary layer. Requirements of fast but smooth and non-oscillating increase of the arc temperature near the cathode and reasonable values of $s$ and $E(s)$ play a role of complementary limiting conditions of the model. Their fulfillment can be ensured by a fine tuning of the state variables in a close vicinity of the global minimum. For this task, a deeper insight into the behavior of individual components of the objective function is useful.

In the following, results are computed for a constant exponent $n_{\mathrm{r}}$ and are given in spatial diagrams and their projection to the $\left[T_{\mathrm{A}}(s) j_{0}\right]$ plane to demonstrate sensitivity of the objective function and its components to the current density at the cathode tip $j_{0}$ and to the arc temperature $T_{\mathrm{A}}(s)$ at the end of the cathode boundary layer. The chosen exponent $n_{\mathrm{r} \_\min }=2.45$ corresponds to the global minimum of the objective function $\Delta_{\text {tot_min }}$ for the investigated experiment.

In spatial diagrams, rather sharp shapes in Fig. 3 $(\mathrm{a}, \mathrm{b})$ clearly indicate that the first two components of the objective function, which express fidelity of the computed arc power loss distribution in the input and main parts of the anode channel in comparison with the measured power loss, are highly sensitive especially to the current density $j_{0}$ at the cathode tip. As far as the arc temperature $T_{\mathrm{A}}(s)$ at the end of the 


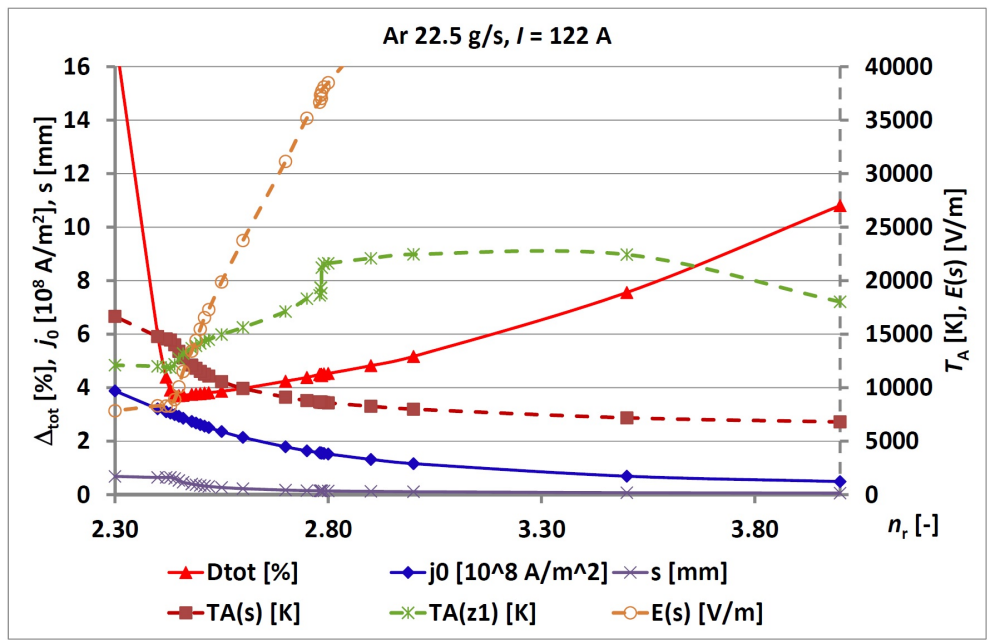

Figure 1. Minimum values of the objective function $\Delta_{\text {tot }}$ together with the corresponding values of the current density at the cathode tip $j_{0}$, the boundary layer width $s$, the arc temperature $T_{\mathrm{A}}(s)$ and the electric field intensity $E(s)$, both at the end of the boundary layer, and the arc temperature $T_{\mathrm{A}}\left(z_{1}\right)$ at the first step $z_{1}=1 \mathrm{~mm}$ vs. the exponent $n_{\mathrm{r}}$, for the investigated experiment.
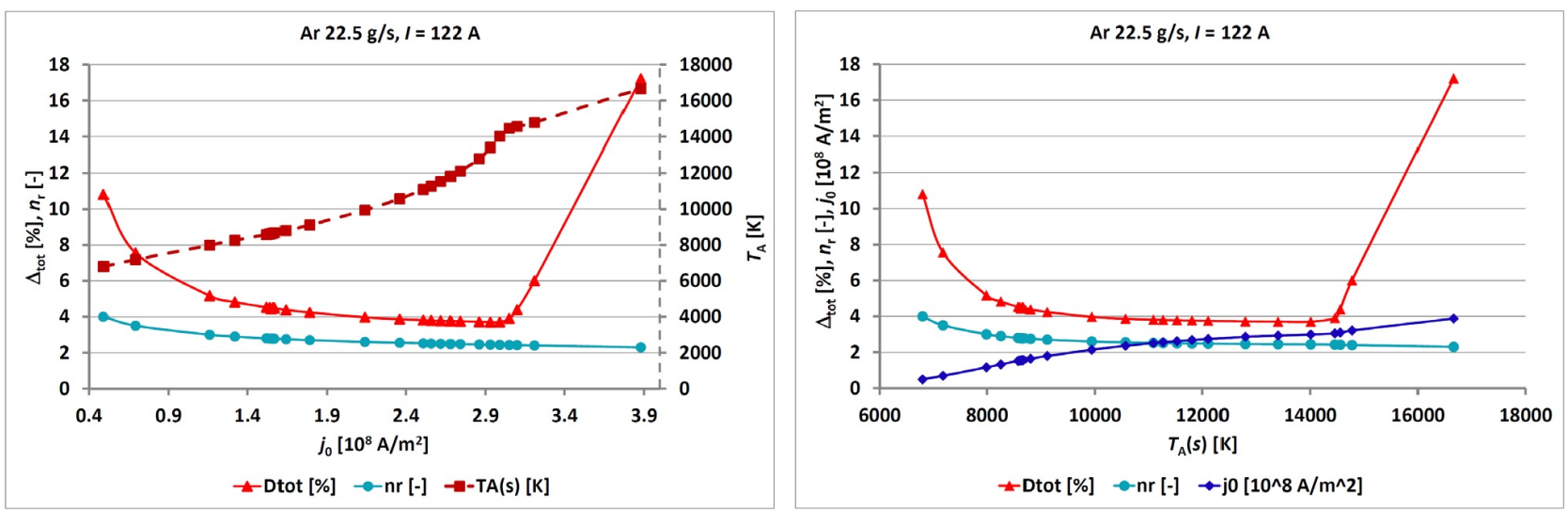

Figure 2. Minimum values of the objective function $\Delta_{\text {tot }}$ together with the corresponding values of the exponent $n_{\mathrm{r}}$ and the arc temperature $T_{\mathrm{A}}(s)$ at the end of the boundary layer vs. the current density at the cathode tip $j_{0}$ (left), and together with the corresponding values of the exponent $n_{\mathrm{r}}$ and the current density at the cathode tip $j_{0}$ vs. the arc temperature $T_{\mathrm{A}}(s)$ at the end of the boundary layer (right), for the investigated experiment.

cathode boundary layer is concerned, obviously $\Delta_{\text {in }}$ exhibits the highest sensitivity to this state variable; this observation is in accordance with preliminary expectations. Both $\Delta_{\text {in }}$ and $\Delta_{\text {ch }}$ change their sign in the tested range. In other words, their minimum absolute value is zero. This fact is stressed in Fig. 3 $(\mathrm{d}, \mathrm{e})$.

The spatial diagram of $\Delta_{\mathrm{U}}$ in Fig. 3 (c) is given in fine resolution (values between $2 \%$ and $4 \%$ ) and is much flatter. Finally, the spatial diagram of $\Delta_{\text {tot }}$ in Fig. 3 (f) is steep and its lowest values correspond to the intersection of $\Delta_{\text {in }}=0$ and $\Delta_{\text {ch }}=0$ lines (Fig. 3 d,e). It can be concluded that the behavior of $\Delta_{\text {in }}, \Delta_{\text {ch }}$ is crucial for reaching the minimum of the objective function while the third component $\Delta_{\mathrm{U}}$ has a predominant influence on the reached minimum value of $\Delta_{\text {tot_min }}$.

As can be seen from Fig. 1, in the global minimum of $\Delta_{\text {tot min }}$ the temperature $T_{\mathrm{A}}(s)$ is higher than the temperature $T_{\mathrm{A}}\left(z_{1}\right)$. Obviously, the requirement of the increasing arc temperature $T_{\mathrm{A}}(z)$ near the arc beginning is not met in the found global minimum of the objective function in this case. Solving this problem only by a shift of $T_{\mathrm{A}}(s)$ to lower values while keeping the found $n_{\mathrm{r}}$ unchanged would significantly rise $\Delta_{\text {tot }}$, especially due to $\Delta_{\text {in }}$. Fortunately, close above $n_{\mathrm{r} \_ \text {min }}=2.45$ the curves $T_{\mathrm{A}}\left(s, n_{\mathrm{r}}\right)$ and $T_{\mathrm{A}}\left(z_{1}, n_{\mathrm{r}}\right)$ intersect, thus a slight shift above this intersection, to a neighboring minimum with higher $n_{\mathrm{r}}$ can ensure the required relation between $T_{\mathrm{A}}(s)$ and $T_{\mathrm{A}}\left(z_{1}\right)$. Of course, the shift out of the global minimum must lead to a worse value of the objective function, but the increase of $\Delta_{\text {tot }}$ is of the order of low tenths of percent and is acceptable. On the other hand, a steep increase of $E(s)$ over physically reasonable values does not allow to retreat too far from the global minimum (Fig. 1).

In the discussed case, the global minimum of $\Delta_{\text {tot_min }}=3.705 \%$ is found for the following values: $n_{\mathrm{r} \_ \text {min }}=2.45, j_{0 \_ \text {min }}=2.93 \times 10^{8} \mathrm{Am}^{-2}, T_{\mathrm{A}}(s)_{\min }=$ 


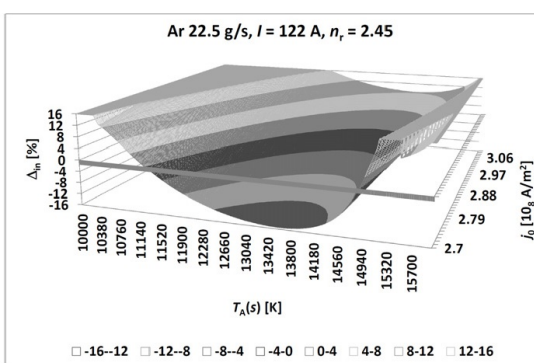

(a)

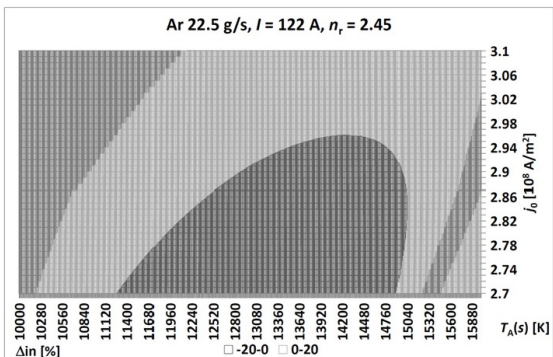

(d)

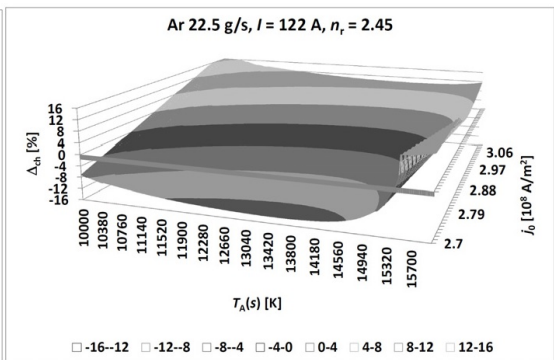

(b)

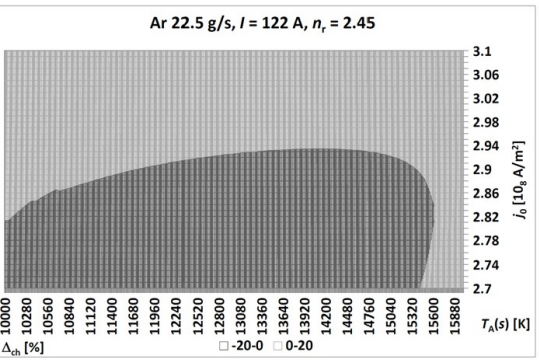

(e)

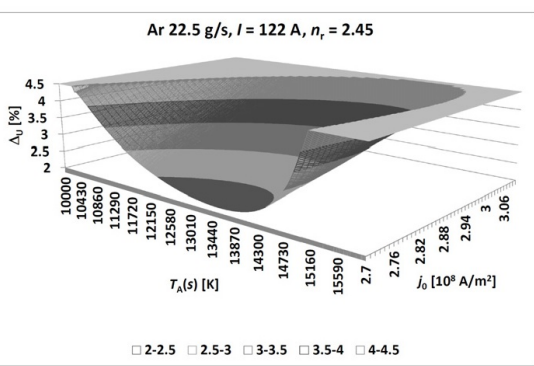

(c)

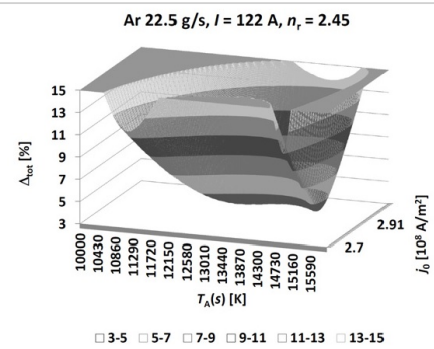

(f)

Figure 3. The computed values for the investigated experiment and exponent $n_{\mathrm{r}}=2.45$ corresponding to the obtained global minimum of the objective function: individual components of the objective function $\Delta_{\mathrm{in}}(a, d), \Delta_{\mathrm{ch}}(b, e)$ and $\Delta_{\mathrm{U}}(c)$, the objective function $\Delta_{\text {tot }}$ as a whole (f), vs. the current density $j_{0}$ (in the range of $(2.7-3.1) \times 10^{8} A m^{-2}$ ) and the arc temperature $T_{\mathrm{A}}(s)$ (in the range (10-16) kK).

$13400 \mathrm{~K}$. The optimum solution for which the smooth increase of the arc temperature near the beginning and a reasonable value of $E(s)$ are ensured while only negligible deterioration of $\Delta_{\text {tot_opt }}=3.765 \%$ is obtained is the following: $n_{\mathrm{r} \_ \text {opt }}=2.49, j_{0 \_ \text {opt }}=$ $2.68 \times 10^{8} \mathrm{Am}^{-2}, T_{\mathrm{A}}(s)_{\mathrm{opt}}=11810 \mathrm{~K}$.

\section{Conclusions and Further Work}

The paper deals with the sensitivity of the model of intensively blasted electric arc burning in argon inside the arc heater's anode channel to exponent $n_{\mathrm{r}}$ and the current density $j_{0}$ at the cathode tip, which define the arc column development, and the arc temperature $T_{\mathrm{A}}(s)$ at the end of the near-cathode boundary layer. Such a vector of these three quantities is sought when the objective function $\Delta_{\text {tot }}$ reaches its minimum. Fine tuning is then needed to ensure physically reasonable dependences of the arc temperature and electric field intensity near the arc beginning. Based on typical experimental results, the paper tries to demonstrate quantitatively how significantly changes of $n_{\mathrm{r}}, j_{0}$ and $T_{\mathrm{A}}(s)$ affect the objective function and its components $\Delta_{\mathrm{in}}, \Delta_{\mathrm{ch}}$, and $\Delta_{\mathrm{U}}$. Just the analysis of individual components $\Delta_{\text {in }}, \Delta_{\text {ch }}$, and $\Delta_{\mathrm{U}}$ of the objective function elucidates the behavior of the model as a whole and confirms the correctness and effectiveness of the computation procedure. Utilization of the model with numerous sets of different experimental results is intended to confirm or enhance its applicability.

\section{Acknowledgements}

This research work has been carried out in the Centre for Research and Utilization of Renewable Energy
(CVVOZE). Authors gratefully acknowledge financial support from the Ministry of Education, Youth and Sports of the Czech Republic under OP VVV Programme (project No. CZ.02.1.01/0.0/0.0/16013/0001638 CVVOZE Power Laboratories-Modernization of Research Infrastructure).

\section{References}

[1] J. Senk, I. Laznickova, and I. Jakubova. Estimation of electric arc power loss using net emission coefficient of argon. In Proceedings of EPE 2018. Brno Univ. of Technology, 2018. doi:10.1109/EPE.2018.8396015.

[2] J. Senk, I. Jakubova, and I. Laznickova. Analysis of intensively blasted electric arc burning in the arc heater's anode channel. Acta Polytechnica, 56(5):395-401, 2016. doi:10.14311/AP.2016.56.0395.

[3] J. Senk, I. Jakubova, and I. Laznickova. Updated version of the simplified model of intensively blasted electric arc. Acta Polytechnica, 58(4):264-270, 2018. doi:10.14311/AP.2018.58.0264.

[4] A. Farmer and G. Haddad. Material Functions and Equilibrium Composition of argon and Nitrogen Plasma. Csiro Division of Applied Physics, 1989.

[5] O. Coufal. Composition and thermodynamic properties of thermal plasma up to $50 \mathrm{kk}$. J Phys D Appl. Phys, 40(11):3371-3385, 2007. doi:10.1088/0022-3727/40/11/018.

[6] P. Kloc. Computed values of net emission coefficient of argon. Private communication, 2018.

[7] H. Schlichting. Grenzschicht Theorie. Verlag G. Braun, Karlsruhe, Germany, 1965.

[8] J. Lowke. Predictions of arc temperature profiles using approximate emission coefficients for radiation losses. J Quant Spectrosc Radiat Transfer, 14:111-122, 1974. doi:10.1016/0022-4073(74)90004-1. 